\title{
AN EXAMPLE OF QPvQ
}

\section{Ken Thompson}

[The following contribution by Ken Thompson illustrates chess database results obtainable for various initial positions, of which one has been selected. The initial position is not, it should be noted, the maximin for this configuration (cf. Vol. 9, No. 3, p.136), though it is most illuminating. The text arrangement is as follows. Any indented line represents optimal play, for which the number of moves yet to be made decreases by unity for each complete (two-ply) move. Variations below these indicate the consequences of non-optimal moves, where, e.g., (-19) denotes that Black's response listed has shortened White's winning path by 19 full moves. Occasionally, (0) will occur to state this variation to be equi-optimal (e.g., see Black's 35th move). Conversely, a parenthesised positive number, e.g. $(+2)$, means that White has responded non-optimally, prolonging his own winning path by 2 moves in the example given. Zeroes in this case also represent equi-optimal continuations. - Eds.]



1 bge (56) $\mathrm{ge}_{\mathrm{a} 8 \mathrm{~g}}(56)$

$1 \ldots$ ․ㅏㅁ $2+(-19)$ 쌈 $44(-40)$ 씩3 $(-43)$

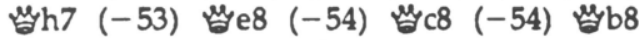
$(-54)$

2 toh 2 (55)

2 bg1 (+2)

\section{2 ... $\mathrm{G}_{\mathrm{g}} \mathrm{ht}(55)$}

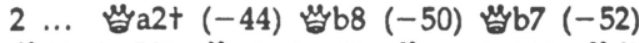

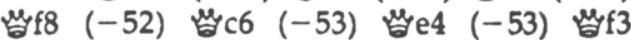
$(-53)$ 쌍ㅇ $(-53)$ 쌍 8 ( $(-53)$ 쌍ㄱ $(-53)$ 쌈a6 (-53) 쌈a4 $(-53)$ 쌈a3 $(-53)$

$3 \mathrm{~g}^{3}$ (54)

3 dg $2(+2)$

3 ... $\mathrm{c} 3+(54)$

$3 \ldots$ ‥

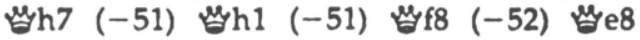
$(-52)$ 쌍ㅇ (-52) क्db2 (-52) क्वa2 (-52) क्do1 (-52)

$4 \mathrm{gg}^{4}$ (53)

4 the $2(+2)$

\section{4 ... 밤 $\mathrm{d} 4+(53)$}

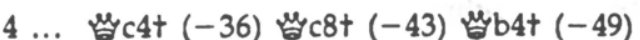

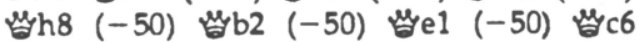

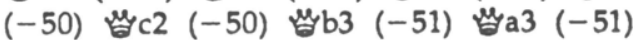
․ㅏㅁ3 (-51) bb2 (-51) कa $(-51)$ bb1 $(-51)$

\section{Hef4 (52) $\mathrm{Hg}_{\mathrm{g}} 7 \mathrm{t}(52)$}

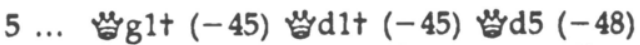



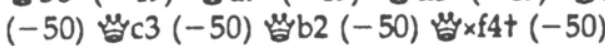

6 bf5 (51)

6 thf (+1) 6 thh5 (+3) 6 bh4 (+3) 6 bh 3 $(+4)$

6 ... 쌉 $88+(51)$

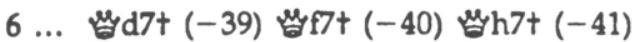

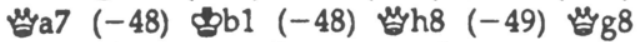

$(-49)$ ․ㅏㅁ $7(-49)$ bb

7 be4 (50)

7 be5 (+1) 7 bg4 (+2) 7 bg5 (+3)

7 ... $\mathrm{Ha8}+(50)$

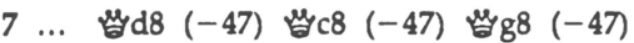

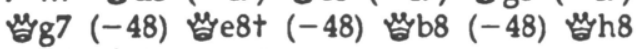
$(-48)$ ․ㅏㅇ $x 4+(-48)$

8 be3 (49)

8 bd3 (+1) 8 bd4 (+1) 8 be5 (+2) 8 bf 5 $(+3)$

8 ... ㅂa3t (49)

$8 \ldots$ …

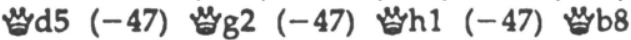

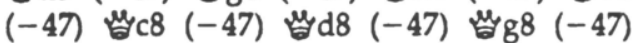

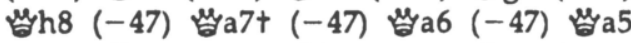
$(-47)$ ్a $(-47)$ कb2 $(-47)$ ba2 $(-47)$ tobl (-47)

9 be2 (48)

9 bd4 (+2) 9 bd2 (+2) 9 be4 (+2) 9 bुf2 $(+5)$

9 ... 쌈b2t (48)

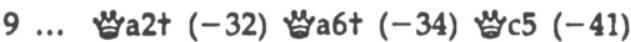

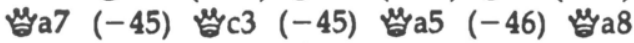

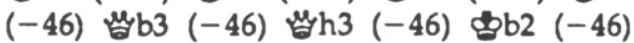
ba2 (-46) bb1 (-46)

10 방ㄹ2 (47)

10 bd $3(+2) 10$ bd $1(+4) 10$ bf $3(+5) 10$ be3 (+5) 10 bf $1(+7) 10$ bel (+7)

10 ... 붕ㄹ (47)

$10 \ldots$ ․ㅏㅁ $5+(-19)$ 쌈 $5+(-20)$ 쌈 $7(-38)$

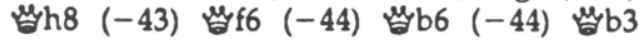

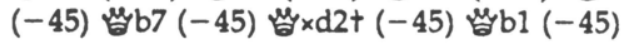

11 be3 (46) 바이 (46)

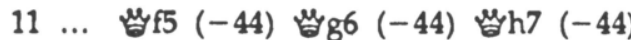

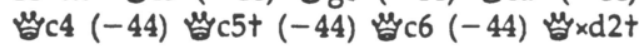
$(-44)$ 
12 쌈 $\mathrm{d} 3$ (45) 쌈 $e 6+(45)$

$12 \ldots$ ․ㅏㅁ $b 6+(-43)$ kㅏㅁ $\times \mathrm{d} 3+(-43)$

13 bf4 (44)

13 d $2(+2) 13$ bf $2(+2) 13$ की $3(+2)$

13 ... 쌈 $\mathrm{f} 6+(44)$

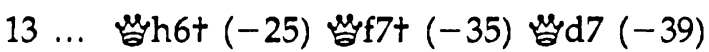
쌈으 $(-42)$

14 b4 (43)

14 bg3 (+2) 14 be3 (+4) 14 be4 (+5)

14 ... 쌈 $77+(43)$

$14 \ldots$ ․ㅏㅁ $66+(-20)$

15 bh5 (42)

15 fo $3(+2) 15$ th $3(+2) 15$ tof (+2) 15 bh $4(+2)$

15 ... 쌈 $\mathrm{f} 7+$ (42)

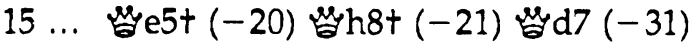
16 참 $g 6$ (41)

16 gg $4(+2) 16$ th6 (+2) 16 gg $(+2) 16$ bh $4(+2)$

$16 \ldots$ ‥ $\mathrm{f} 3+(41)$

$16 \ldots$ 쌈 $\mathrm{d} 5+(-26)$ 쌈 $\mathrm{d} 7(-32)$ 씸 $\mathrm{b} 7(-38)$

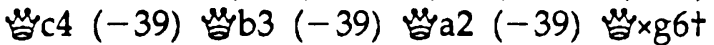
$(-39)$ 쌈a7 (-39) 쌈f4 (-39) 쌈f2 (-39) 쌉f1 $(-39)$

17 bh4 (40) ․ㅏㅁ $\mathrm{f4t}(40)$

$17 \ldots$ kㅏㅁ $\mathrm{f} 2+(-19)$ 쌈 $\mathrm{h} 1+(-24)$ 쌈3 $(-35)$

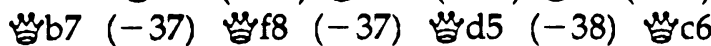

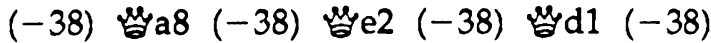
쌈 $\mathrm{e} 3(-38)$ 쌈 b3 $(-38)$ 쌈 $\mathrm{a} 3(-38)$ 쌈 $\mathrm{f} 1$ $(-38)$ bb2 $(-38)$ ba $2(-38)$

18 bh3 (39) ․ㅏㅇ f1t (39)

$18 \ldots$ ․ㅏㅁ $3+(-3)$ kㅏㅁ $\mathrm{f} 3+(-3)$ kㅏㅇ $\mathrm{d} 4(-30)$

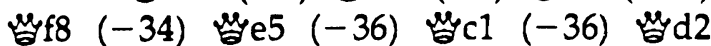

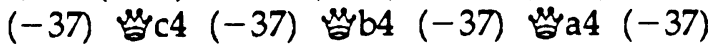
쌈 $\mathrm{f} 2(-37)$

19 쌍 82 (38)

19 bh4 (+2) 19 bh2 (+2)

19 ... 쌉 $\mathrm{f5}+(38)$

$19 \ldots$ ․ㅏㅁ $\mathrm{d} 3+(-13)$ 쌍 $\mathrm{f} 6(-26)$ kㅏㅁ $\mathrm{d} 1(-27)$

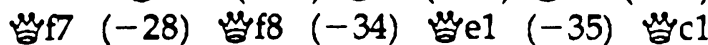
$(-35)$ 쌈 b1 $(-35)$ 쌈c4 $(-36)$ 쌈 b5 $(-36)$ kㅏㅂa6 $(-36)$ kㅏㅁ $\times \mathrm{g} 2+(-36)$ 쌈 $\mathrm{f} 4(-36)$

20 bh2 (37) 쌍 h5t (37)

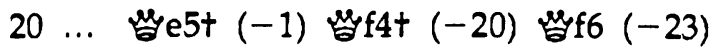

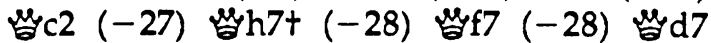

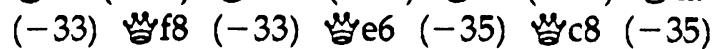

쌈 $\mathrm{d} 3(-35)$ 쌈 $\mathrm{b} 1 \quad(-35)$ 씸 $\mathrm{c} 5(-35)$ 쌈 $\mathrm{b} 5$ $(-35)$ 참 $a 5(-35)$ bb1 $(-35)$

21 쌍 33 (36) 창 $\mathrm{e} 5+(36)$

$21 \ldots$ 참 $\mathrm{f7}(-30)$ 쌈 $\mathrm{e} 8(-30)$ 쌈 $\mathrm{b} 5(-30)$

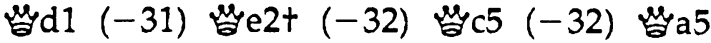

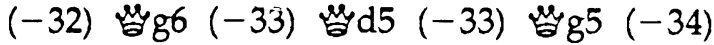
쌈 $\times \mathrm{h} 3+(-34)$

22 kㅏㅁ 3 (35) 쌍 $h 5+(35)$

$22 \ldots$ ․ㅏㅁ $2+(-1)$ 쌈f6 $(-19)$ 씸 $8+(-19)$

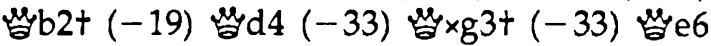
$(-33)$ 쌈 $\mathrm{e} 8(-33)$ kㅏㅁ $\mathrm{d} 5(-33)$ 씩ㄷ $(-33)$

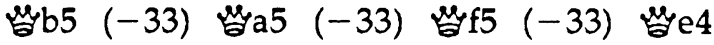
$(-33)$

\section{3 gi (34)}

23 bg $2(0) 23$ 쌈 $\mathrm{h} 3(+2)$

\section{3 ... ․ㅏㅁ $c 5 t(34)$}

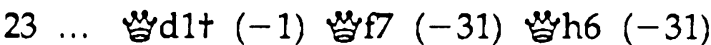


$(-32)$ ํㅏㄹ $(-32)$ kff5 $(-32)$ kfd5 $(-32)$

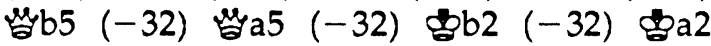
$(-32)$

24 ge (33)

24 bf 1 (0) 24 bh2 (+2) 24 bh1 (+2)

24 ... ‥ $\mathrm{d} 5+(33)$

$24 \ldots$ kㅏㅁ $22+(-10)$ kㅏㅁ $66+(-22)$ kga7 $(-30)$

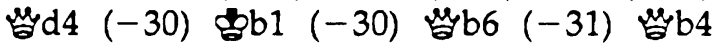

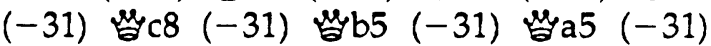

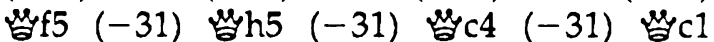
$(-31)$ bb2 (-31) ba2 $(-31)$

25 fif2 (32)

25 bf $1(+1) 25$ bg1 (+2) 25 कh 3 (+3) 25 bh2 $(+3)$

\section{$25 \ldots$... $\mathrm{d} 44+(32)$}

$25 \ldots$... b2 $(-13)$ kct5t $(-14)$ ba2 $(-14)$ bb1 (-14) 쌈a2t $(-15)$ 쌈 $f 5 t(-15)$ 쌈 $77 t$

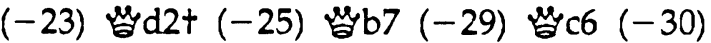

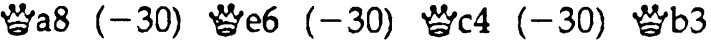

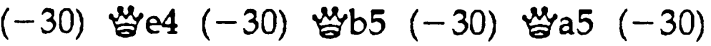
쌈 $\mathrm{h} 5(-30)$ 씸 $\mathrm{d} 1(-30)$

26 be2 (31)

26 be1 (+1) 26 bf $3(+2) 26$ bg2 (+2) 26 bf $1(+2)$

$$
26 \text {... kㅏㅁ } 4+(31)
$$

$26 \ldots$... b2 $(-11)$ 쌈 $c 4+(-14)$ 쌈 $b 2+(-16)$



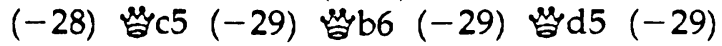
쌈 b4 (-29) 쌈 a4 $(-29)$ bुb1 $(-29)$ 
$27 \mathrm{~d} 2(30)$

$27 \mathrm{~d} 1(+2) 27$ ff2 $(+2) 27 \mathrm{ff} 1(+3)$

27 ... ․ㅏㅁ $\mathrm{d} 4+(30)$

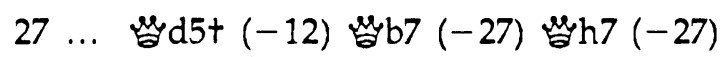

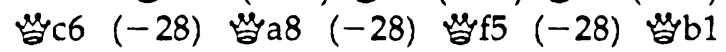



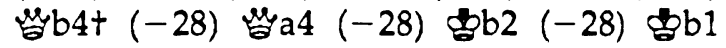
$(-28)$

28 낭 $\mathrm{d} 3(29)$

28 be2 $(+2) 28$ be1 $(+3)$

28 ... 빵 b2t (29)

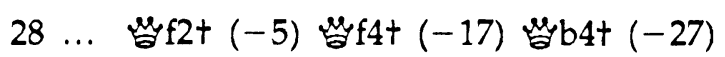
랑 $\times \mathrm{d} 3+(-27)$

\section{9 be1 (28)}

29 kㅏㄱㄹ (+2) 29 be3 (+2) 29 bd $1(+19)$

29 ... ․ㅏㅁ b4t (28)

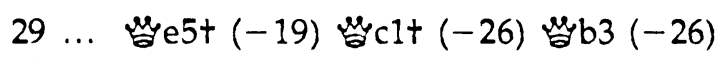

쌍ㄷㄹ $(-26)$

30 bf 1 (27)

30 f2 $(+2) 30$ be $2(+2) 30$ d1 $(+20)$

$30 \ldots$ …의 $\mathrm{f} 4+(27)$

$30 \ldots$ bb2 $(-24)$ 쌈b3 $(-25)$

31 be2 (26)

31 be1 (+2) 31 bg2 (+8) 31 bg1 (+11)

31 ... 창 h2t (26)

$31 \ldots$ kㅏㅁ $g 4+(-9)$ kㅏㅁ $5+(-17)$ bb2 $(-24)$

32 be1 (25)

32 d 1 (0) 32 bf $1(+2) 32$ f $3(+21) 32$ be $3(+22)$

$32 \ldots$ kh $\mathrm{h} 4+(25)$

$32 \ldots$ kㅏㅁ $1+(-9)$ kㅏㅁ $\mathrm{h} 1+(-9)$ 창 $\mathrm{e} 5+(-16)$

땀 $2(-23)$ b $\mathrm{b} 2(-23)$

$33 \mathrm{~d} 2$ (24)

33 be $2(+2) 33$ d $1(+5) 33$ bf $1(+22)$

33 ... ․ㅏㅁ $\mathrm{f} 2 \mathrm{f}(24)$

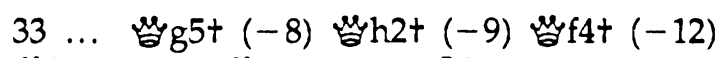

khbt $(-16)$ kb $4+(-22)$ bb2 $(-22)$

34 bc3 (23)

34 bd1 (+2) 34 kुge2 (+7) 34 bc1 (+25)

34 ... ․ㅏㅁ $c 5+(23)$

$34 \ldots$ kㅏㅁ $\mathrm{b} 2+(-9)$ 창 $\mathrm{e} 1+(-19)$ kㅏㅁ $\mathrm{f} 6+(-20)$

35 남 44 (22)

$35 \mathrm{~d} 2(+2) 35$ bb3 (+27)
35 ... kㅏㅇ a3t (22)

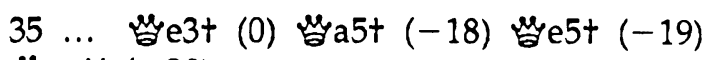
참 $\times c 4+(-20)$

36 bc2 (21) 쌈 $c 1+(21)$

$36 \ldots$ ․ㅏㅁ $2+(-1)$

37 d 3 (20)

37 bुb3 (+1)

37 ... 쌈 $\mathrm{d} 1+(20)$

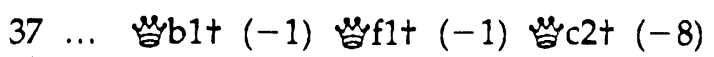
쌈 $\mathrm{a} 3+(-18)$ 쌈 $\times \mathrm{c} 4+(-18)$

38 bु (19) ․ㅏㅁ $\mathrm{e} 1+(19)$


bib1 $(-17)$

39 bd4 (18)

39 d3 $(+2) 39$ b3 $(+3) 39$ bc $(+3)$

39 ... 창 $11+(18)$

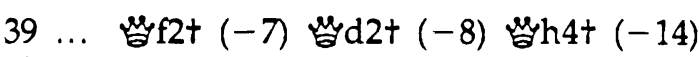

kㅏㄹ 8 ( $(-15)$ kㅏㅁ $\mathrm{d} 1+(-15)$ kㅏㅁ $\mathrm{g} 3(-16)$

40 bd5 (17)

40 be4 (+1) 40 be5 (+2) 40 bd3 (+3) 40 bc3 $(+5)$

40 ... 참 $88+(17)$

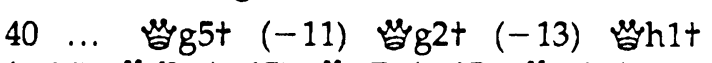

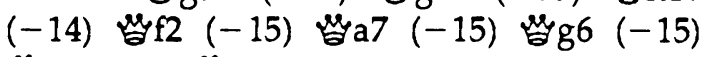
kㅏㅂ. $7(-15)$ kㅏㅁ $\mathrm{d} 1+(-15)$

41 bc5 (16)

41 bd $4(+2)$

41 ... 씀 $\mathrm{c} 8+(16)$

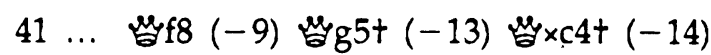

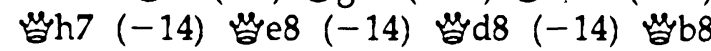

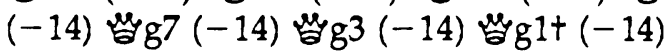

42 bd4 (15)

42 b4 (+2) 42 bb5 (+2) 42 d5 (+2)

42 ... ․ㅏㅁ $h 8+(15)$

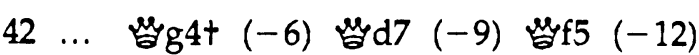

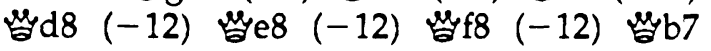
$(-13)$ 칙 $\mathrm{h} 3(-13)$ kㅏㅁ $\times \mathrm{c} 4+(-13)$

43 d3 (14)

43 b5 (+2) 43 be4 (+2) 43 bd5 (+3)

43 ... 낭 $h 3+(14)$

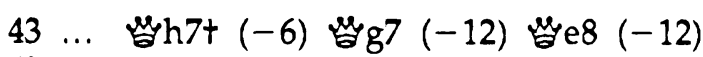
kydg $8(-12)$ bb2 (-12) tb1 $(-12)$

44 dc2 (13)

44 d4 (+2) 44 be4 (+5) 44 bd2 (+9) 
44 ... 창 $22+(13)$

$44 \ldots$ kgh $2+(-1)$ k火g $55+(-2)$ kegh7t $(-5)$ 쌉e6 $(-11)$ 씸 $77(-11)$

45 bुb3 (12)

$45 \mathrm{~d} 3(+7) 45 \mathrm{bc} 3(+8)$

45 ... ․ㅏㅁ b7t (12)

$45 \ldots$ 쌈 $22+(-1)$ 쌈 $2+(-2)$ 쌈 $a 2+(-2)$

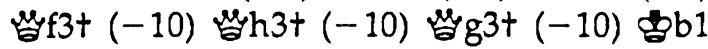
$(-10)$

46 ba3 (11)

46 ba4 (+2) 46 쌈b4 (+4) 46 bc3 (+9) 46 bc $2(+10)$

46 ... 쌈 b2t (11)

$46 \ldots$ 쌈 $\mathrm{f} 3+(-3)$ kㅏㅁ $a 7+(-3)$ kㅏㅁa8t $(-8)$ kfb1 (-9)

47 ba4 (10) kㅏㅁ $\mathrm{c} 2+(10)$

$47 \ldots$ kㅏㅁ $\mathrm{d} 2(-4)$ kㅏㅁ $77(-7)$ 쌈 b7 (-8) 쌈 b1 $(-8)$

48 bb5 (9)

48 b4 (0)

$48 \ldots$ ․ㅏㅁ b2t (9)

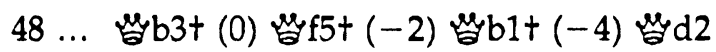


$(-7)$

49 bc6 (8)

49 bc5 (0) 49 ํㅏㅁ $4(+1) 49$ ba $(+2)$

49 ... 쌍 $c 2(8)$

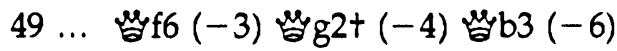

50 6c5 (7)

50 kㅏㅁ $5(+1) 50$ bb5 (+2) 50 bd5 (+5)

$50 \ldots$ ․ㅏㅇ $\mathrm{f} 5+(7)$

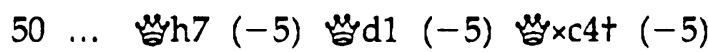

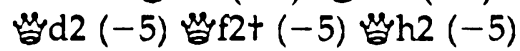

51 bb4 (6)

51 bb6 (+1) 51 kㅏㅁ $5(+2) 51$ d4 (+5) 51 bc6 $(+12)$

$51 \ldots$ …

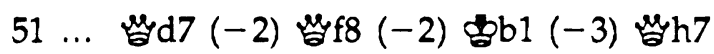

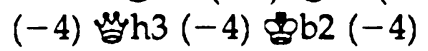

52 ba5 (5)

$52 \mathrm{bc5}(+2) 52$ 씸 $3(+3) 52 \mathrm{bc}(+15)$

$52 \ldots$ …랄 $1+(5)$

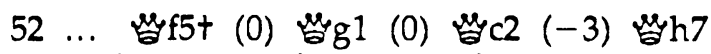
$(-3)$ 쌈 b2 $(-3)$ 쌈 b3 $(-3)$ 쌈 7 ( $(-3)$ 쌈 b8 $(-3)$ kㅏㅁ $11(-3)$
53 कa6 (4)

53 bb6 (+3) 53 kूb4 (+4) 53 bb $5(+5)$

53 ... 쌍 $\mathrm{d} 1$ (4)

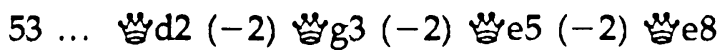
$(-2)$

$54 \mathrm{~kg}$ c3t (3)

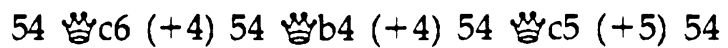
쌈ㄱ $(+7) 54$ ․ㅏㅁf4 (+9)

54 ... bb1 (3)

$54 \ldots$ ba2 (0)

55 남 6 (2)

55 쌈 $44+(+1) 55$ kㅏㅇ $5(+1) 55$ 쌍 $5(+1) 55$

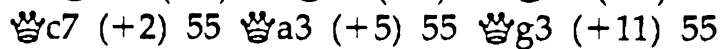

쌈 $\mathrm{f} 6(+12)$

55 ... ․ㅏㅇ $\mathrm{d} 3+(2)$

$55 \ldots$ ․ㅏㅁ $\mathrm{e} 2+(0)$ ․ㅏㅁ $\mathrm{f} 1+(0)$

56 쌈b5t (1)

56 bb7 (+1) 56 ba7 (+2) 56 bb6 (+2) 56 ba $(+3)$

56 ... bc1 (1)

$56 \ldots$ ․ㅏㅁ $\times b 5+(+1)$

$57 \mathrm{~d} 7$ (5) 